Research Article

\title{
Trotting Motion of the Quadruped Model with Two Spinal Joints and Its Dynamics Features
}

\author{
Zhang $\operatorname{Li} \oplus^{1}$ and Yuegang Tan ${ }^{2}$ \\ ${ }^{1}$ China Nuclear Operation Technology Corporation, Ltd., Donghu New Technology District, Wuhan 430070, China \\ ${ }^{2}$ School of Mechanical and Electronic Engineering, Wuhan University of Technology, Wuhan 430070, China \\ Correspondence should be addressed to Zhang Li; lz2015@whut.edu.cn
}

Received 17 December 2019; Revised 15 May 2020; Accepted 23 May 2020; Published 22 June 2020

Academic Editor: Oscar Reinoso

Copyright ( $) 2020$ Zhang Li and Yuegang Tan. This is an open access article distributed under the Creative Commons Attribution License, which permits unrestricted use, distribution, and reproduction in any medium, provided the original work is properly cited.

\begin{abstract}
The spine plays important roles in the quadruped locomotion. To investigate the effects of the spine on the quadruped trotting motion, firstly, a sagittal passive model is proposed which contains four massless springy legs and two passive spinal joints. To generate the trotting gait of the model, the multibody hybrid dynamics model is established based on the defined events. The combination of optimization tools is used to find the suitable solution space in which the model can maintain a periodic motion. It reveals that the quadruped trotting motion results from the coordinated features of the spine and the legs. By comparing the model with the rigid body, it is proven that the spinal joints can reduce the effect of the ground reaction forces on the body in a special velocity range. Then, a hybrid controller whose objective is to maintain the kinematic coordination between the spinal joints is applied and it replaces the passive spinal joints, and the results prove that it can make the model achieve a stable periodic motion. Finally, the prototype of the quadruped robot with two spinal joints based on the model is established and its trotting motion is achieved successfully. The experiment results also indicate the compliant effect of the spine on the motion performance. Consequently, the effects of the spine at trotting gait are helpful to guide the development of the quadruped robots.
\end{abstract}

\section{Introduction}

The mammals have the flexible motion ability to adapt to the environments, which benefits from the coordination mechanisms, including the levels of neural control, muscle function, interlimb coordination, intersegmental coordination, and intuitive motion mechanism [1]. Studying the dynamic features of the legged motion on the level of intuitive motion mechanism has always been a hot research topic in both biology and robotics areas.

A general finding reveals that when the humans or mammals run, the legs act as springs and experience a compression and extension process to improve the energy efficiency and the compliance of the interaction with the ground [2]. Accordingly, a general model template, Spring Loaded Inverted Pendulum (SLIP), was proposed [3] and extended [4-7]. To analyze the intuitive motion mechanism of quadruped motion at different gaits, the planar model including a rigid body and four massless springy legs was established based on the SLIP model [8].

Although this model basically represents the dynamic modes of the quadruped motion, it does not take the spine into account. Many studies found out that the spine plays an important role in the quadruped motion $[9,10]$. Therefore, some researchers added the spine element into the models to investigate the effects of the spine on the motion performance. Some research studies have revealed that the model with a spinal joint has the advantages of decreasing the cost of transport [11], optimizing the energy distribution [12, 13], decreasing the ground reaction forces (GRFs) [14-16], and improving the stability [17] and maneuverability [18]. In addition, the number of the spinal joints in the quadruped motion also has important effects on the motion performance [19].

In this paper, we establish a planar passive model with two spinal joints to investigate the effects of the spine on the 
dynamic performances of quadruped trotting motion. The Poincaré Mapping and combined optimization method are used to search for the suitable parameters and generate the periodic trotting gait. By comparing the model with the rigid body, the effects of the spinal joints are found and discussed. Then, the active control is applied and replacing the passive spinal joints to analyze the effect of the coordination between two spinal joints appeared in the passive trotting motion. In this process, a hybrid controller based on the motion of the spinal joints is applied, which proves that coordination between the spinal joints has the effect of improving the stability of the passive model. Finally, the real quadruped robot with two spinal joints is established. Some experiments for the trotting motion show the effect of the two spinal joints on the ground reaction forces.

\section{Methods}

2.1. Quadruped Model with Two Spinal Joints. First, we propose a planar quadruped dynamic model with two passive spinal joints, which is restricted in the sagittal plane, as shown in Figure 1. Four massless springs inspired from the mammals [2] are used to act as the legs (forelimb stiffness $k_{f}$ and hindlimb stiffness $k_{h}$, the same initial length $l_{0}$ ). The legs are marked as $\{1,2,3,4\}$ to represent the front left leg, front right leg, hind left leg, and hind right leg, respectively. $\gamma_{i}$ indicates the elevation angle of the leg relative to the vertical direction during the stance phase. Because the legs are massless, there are no dynamics effects for the legs during swing phase. The attack angles are predefined (forelimb $\alpha_{f}$, hindlimb $\alpha_{h}$ ). That is, the legs will reach the attack angle to prepare for standing on the ground. A parameter of swing period $t_{s}$ is defined to avoid the situation where the legs will touch the ground more than once. It is assumed that there is no slid of the foot relative to the ground.

The spine can be divided into two parts, thoracic and lumbar vertebrae. The thoracic and lumbar joint can simulate the motion of spine on the condition that the model will not be more complex. The spinal joints are defined as passive joints (front joint $k_{s f}$, hind joint $k_{s h}$ ). As a result, the stable motion of the model will result from the nonlinear elastic oscillations. The CoM is located at the geometrical center of each part. The mass and inertia of the front, middle, and hind body are $\left(m_{f}, m, m_{h}\right)$ and $\left(I_{f c}, I_{m c}, I_{h c}\right)$. The legs are attached to the center of mass of the front and hind body.
The lengths of geometrical center of each part to the spinal joint are $l_{f}, l_{m}$, and $l_{h}$.

2.2. Dynamics Model. We use the Lagrange method to establish the dynamics equations of the quadruped model. The generalized coordinate vector of the model is defined as $\mathbf{q}=$ $\left[x, z, \varphi_{m}, \varphi_{f}, \varphi_{h}\right]^{\mathrm{T}} \in \mathrm{Q}$. $\mathrm{Q}$ is the configuration space. $(x, z)$ indicates the coordinate of the center of mass of the middle body. $\varphi_{m}, \varphi_{f}$, and $\varphi_{h}$ refer to the pitch angle of the middle body, front body, and hind body, respectively. These five independent parameters describe the dynamic motion of the model. The coordinate of the center of mass of the front body $\left[x_{f}, z_{f}\right]$ and hind body $\left[x_{h}, z_{h}\right]$ can be stated from generalized coordinates. The dynamic equations of the motion were derived by the Lagrange method as the following form:

$$
\mathbf{M}(\mathbf{q}) \ddot{\mathbf{q}}+\mathbf{V}(\mathbf{q}, \dot{\mathbf{q}})=\mathbf{B}(\mathbf{q}) \cdot \mathbf{u}+\mathbf{J}^{T}(\mathbf{q}) \cdot \mathbf{F},
$$

where $\mathbf{M}(\mathbf{q})$ indicates the mass matrix. $\mathbf{V}(\mathbf{q}, \dot{\mathbf{q}})$ indicates the generalized torque caused by the centrifugal, Coriolis, gravitational force, and spinal elastic force. $\mathbf{B}(\mathbf{q}) \mathbf{u}$ indicates the generalized input torque. $\mathbf{J}^{T} \mathbf{F}$ represents the generalized torque caused by the elastic legs. The elastic force of each leg, which also reflects the GRFs, acts on the model along the axis of the legs.

The system state variables are composed of continuous state $\mathbf{x}$ and discrete state dis. The continuous state $\mathbf{x}$ contains the generalized coordinates and their derivatives. The continuous state space equations of the model with two spinal joints can be obtained from the dynamic equations and given as the following form:

$$
\begin{aligned}
\dot{x} & =\left[\begin{array}{c}
\dot{\mathbf{q}} \\
\mathbf{M}^{-1}(\mathbf{q})\left[-\mathbf{V}(\mathbf{q}, \dot{\mathbf{q}})+\mathbf{J}^{T}(\mathbf{q}) \cdot \mathbf{F}+\mathbf{B}(\mathbf{q}) \cdot \mathbf{u}\right]
\end{array}\right] \\
& =: f(\mathbf{x})+g(\mathbf{x}) \mathbf{u},
\end{aligned}
$$

where the second item at the right side is equal to zero. Since the model is passive and there is no impact occurring when the legs stand on the ground, the continuous states have no abrupt changes, and the dynamics model is a conservative system. The discrete states dis $\left(\left[\text { phase, } f t d_{x}\right]^{T}\right)$ at any time contain two elements: phase is used to identify which phase each leg is in (prepare for touchdown by $\{1\}$, stance phase by $\{2\}$, and swing phase by $\{3\})$ and $f t d_{x}$ is used to record the touchdown position of each leg. Therefore, the state space of the model can be described as the following form:

$$
T Q:=\left\{\left(\mathbf{q}^{T}, \dot{\mathbf{q}}^{T}, \text { phase }, \mathbf{f t d}_{x}\right) \mid \mathbf{q} \in Q, \dot{\mathbf{q}} \in R^{\operatorname{dim}(q)}, \text { phase }_{i} \in\{1,2,3\}, \mathbf{f t d}_{x} \in R^{4}\right\}
$$

2.3. Gait Generation. The gait generation is implemented by using and extending the framework proposed by Remy et al. [20] due to its universality to different gaits where there are different numbers of legs on the ground simultaneously. In the motion simulation, the states of the model are monitored all the time by the following event detection functions to judge whether there is a transition between the phases of the legs:

$$
\left\{\begin{array}{l}
e_{i, 1}=l_{0} \cos \left(\alpha_{f, h}\right)-z_{f, h} \\
e_{i, 2}=l_{i}-l_{0} \\
e_{i, 3}=t-t_{s i}
\end{array}\right.
$$

where $e_{i, j}$ refers to the event detection function of the leg $i$ $(i=1,2,3,4)$ in the phase $j(j=1,2,3) . \alpha_{f, h}$ refers to the attack angle of forelimb leg or hindlimb leg. $l_{i}$ indicates the length 


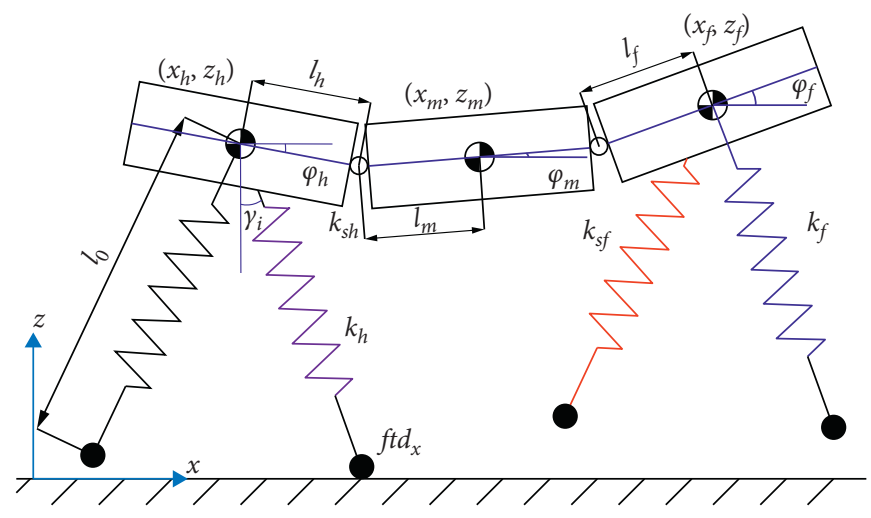

FIgURE 1: Quadruped model with two spinal joints.

of the leg in the stance phase. $t$ refers to the duration that the model has experienced from the initial moment. $t_{s i}$ indicates the predefined moment when the leg finishes the swing phase and enters the phase of preparing for touchdown. Once an event is monitored by the event detection functions, the corresponding leg enters the next phase, and the discrete states are updated by the following state updating functions:

$$
\left\{\begin{array}{l}
\Delta_{i, 1}: \text { phase }_{i}^{+}=2 ; \text { ftd }_{x i}^{+}=x_{f, h}+l_{0} \sin \left(\alpha_{f, h}\right) ; \delta_{i}^{+}=1, \\
\Delta_{i, 2}: \text { phase }_{i}^{+}=3 ; t_{s i}^{+}=t+t_{s} ; \delta_{i}^{+}=0 \\
\Delta_{i, 3}: \text { phase }_{i}^{+}=1,
\end{array}\right.
$$

where $\Delta_{i, j}$ refers to the state updating function of the leg $i$ $(i=1,2,3,4)$ transiting from the phase $j(j=1,2,3)$ to the next phase. Phase ${ }^{+}$indicates the phase of the leg updated. $\boldsymbol{\delta}=\left[\delta_{1}, \delta_{2}, \delta_{3}, \delta_{4}\right]$ is used to identify whether the legs are in the stance phase (stance $\delta_{i}=1$, swing $\delta_{i}=0$ ). Then, the hybrid dynamics equations based on the defined events are given as

$$
\sum: \begin{cases}\dot{x}=f(\mathbf{x}), & \mathbf{X}^{-} \notin S, \\ \mathbf{X}^{+}=\Delta\left(\mathbf{X}^{-}\right), & \mathbf{X}^{-} \in S,\end{cases}
$$

where $S:=\{\mathbf{X} \in T Q \mid e=0, \dot{e}>0\} \subset T Q$ indicates the subspace in the state space which meets equation (3). The solving of the hybrid dynamics equations is implemented by the differential equation solving tool of MATLAB-ode45.

2.4. Searching for the Periodic Motion. In order to achieve the periodic trotting motion, the method of Poincaré Mapping is used which was proposed to numerically search for the periodic motion [21]. The periodic motion with a starting state vector $\mathrm{X}$ must meet the following equation:

$$
\mathbf{P}(\mathbf{X}, \mathbf{p})-\mathbf{X}=0,
$$

where state vector $\mathbf{X}$ is the fixed point of Poincare Mapping and the corresponding Poincare section is $S$. $\mathbf{p}$ refers to the system parameters.

For the periodic trotting motion, we define the initial phase $=\left[\begin{array}{llll}3 & 1 & 1 & 3\end{array}\right]^{T}$. The model moves starting from the liftoff event of the leg 1 and leg 4, and the leg 2 and leg 3 are ready to stand on the ground. Since the trotting gait has the symmetrical feature $[22,23]$, we defined the terminal event $e^{*}$ as the liftoff event of the leg 2 . The phase of the terminal moment should be symmetrical to the initial phase, and all legs are in the air. So the initial discrete states $f t d_{x}$ have no influences on the locomotion, and the discrete vector is removed from the state vector $\mathbf{X}$. Then, the state vector $\mathbf{X}$ for searching is reduced to

$$
\mathbf{X}=\left[z, \varphi_{m}, \varphi_{f}, \varphi_{h}, \dot{z}, \dot{\varphi}_{m}, \dot{\varphi}_{f}, \dot{\varphi}_{h}\right]^{T}
$$

In addition, the searched system parameters are given by $\mathbf{p}=\left[k_{f}, k_{h}, k_{s f}, k_{s h}, \alpha_{f}, \alpha_{h}\right]^{T}$ because these parameters are directly related to the dynamic characteristics. Other system parameters are preset and remain unchanged. The total mass of the model $m_{0}=20 \mathrm{~kg}$, the original length of the leg $l_{0}=0.4 \mathrm{~m}$, and the gravitational constant $g \approx 10 \mathrm{~m} / \mathrm{s}^{2}$. The related parameters are listed in Table 1 . The symbol $*$ indicates the solution space of the initial states.

Then, the problem of periodic motion is translated into solving for equation $\mathbf{F}(\mathbf{X}, \mathbf{p})=\mathbf{P}(\mathbf{X}, \mathbf{p})-\mathbf{X}=0$ at different velocities. The solution space consists of eight variables in the state vector $\mathbf{X}$ and six variables in the system parameter vector $\mathbf{p}$. To solve this problem, the optimization method is applied.

The cost function is defined as the norm of the error vector by the following form:

$$
C(\mathbf{X}, \mathbf{p})=\|\mathbf{P}(\mathbf{X}, \mathbf{p})-\mathbf{X}\| \text {. }
$$

First, the particle swarm optimization (PSO) is used as a tool for preliminary searching because it has a lower dependency on the initial states and can randomly select multiple sets of initial states [24, 25]. The accurate searching is solved numerically with the MATLAB optimization tool Global-Search. The method for accurate searching not only possesses the global searching ability, but also has the advantages of fast convergence and high accuracy. The final cost of all simulation results reaches a reasonably low value (10-4 as the norm of the error vector for 8 state variables).

2.5. Comparison with the Model of Rigid Body and the Canine. To investigate the effect of the spinal joints on the quadruped motion, a model with rigid body is used as the baseline comparison, as shown in Figure 2. This model has equivalent system parameters to the model with spinal joints. To reduce 
TABLE 1: Related variables of the model with two spinal joints.

\begin{tabular}{lcc}
\hline Variables & Values & Parameter \\
\hline$\left[m_{f}, m, m_{h}\right]$ & {$[7,6,7](\mathrm{kg})$} & Mass of each part of the body \\
{$\left[I_{f c}, I_{m c}, I_{h c}\right]$} & {$[0.2,0.04,0.2]\left(\mathrm{kg} * \mathrm{~m}^{2}\right)$} & Inertia of each part of the body \\
{$\left[l_{f}, l_{m}, l_{h}\right]$} & {$[0.159,0.076,0.159](\mathrm{m})$} & Half of length of each part of the body \\
{$\left[k_{s}, k_{s h}\right]$} & {$[*, *](\mathrm{N} * \mathrm{~m} / \mathrm{rad})$} & Stiffness of spinal joints \\
{$\left[k_{f}, k_{h}\right]$} & {$[*, *](\mathrm{N} * \mathrm{~m})$} & Stiffness of forelimb and hindlimb leg \\
{$\left[\alpha_{f}, \alpha_{h}\right]$} & {$[*, *](\mathrm{rad})$} & Attack angle of forelimb and hindlimb leg \\
$l_{0}$ & $0.4(\mathrm{~m})$ & Rest length of the legs \\
$t_{s}$ & $0.08(\mathrm{~s})$ & Predefined swing time \\
{$[x, z]$} & {$[*, *](\mathrm{m})$} & Coordinate of the middle body \\
{$\left[\varphi_{m}, \varphi_{f}, \varphi_{h}\right]$} & {$[*, *, *](\mathrm{rad})$} & Pitch angle of each part of the body \\
{$\left[p h a s e_{0}\right]$} & {$[3,1,1,3]$} & Initial phase of each leg \\
{$\left[f t d_{x 0}\right]$} & {$[0,0,0,0]$} & Initial foot position of each leg \\
$\boldsymbol{\delta}_{0}$ & {$[0.1441,-0.1089,-0.5862,-0.3331](\mathrm{m})$} & Initial state coefficient of each leg \\
\hline
\end{tabular}

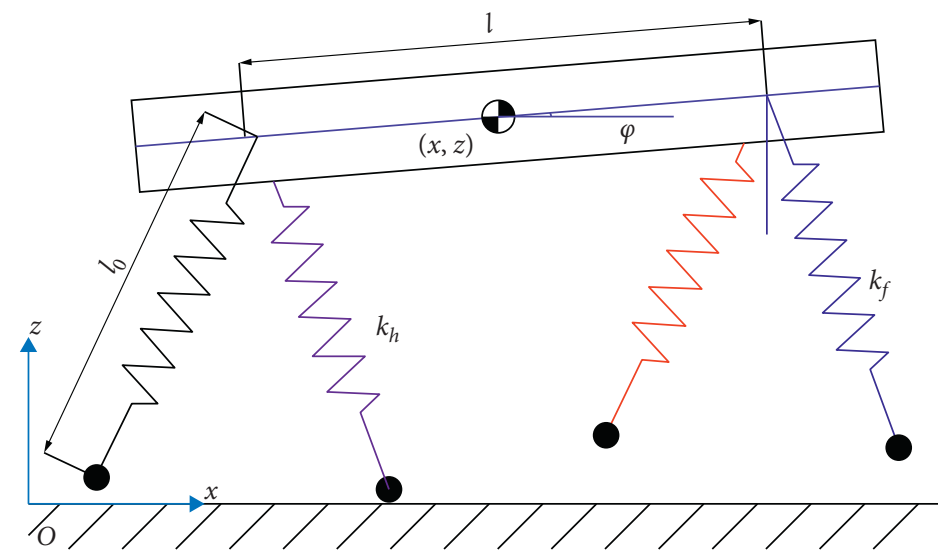

FIGURe 2: Quadruped model with rigid body.

the difference between two models as far as possible, the model with rigid body possesses the same stiffness and attack angle of the legs to the model with two spinal joints.

In the simulation process, we change the velocity to compare the dynamic performances of two models. The biological research found that the trotting gait of the Canine has a range of Froude numbers [26] (1 2.2, by $\left.F_{l}=v /\left(g_{l 0}\right)^{-2}\right)$. So, we choose the velocity range, $0.84 \mathrm{~m} /$ $\mathrm{s} \sim 3.74 \mathrm{~m} / \mathrm{s}\left(F_{l}: 0.42 \sim 1.87\right)$, as the investigating region, which covers a lower velocity range.

First, the stability of periodic motion is compared between two models based on the Floquet theorem [27, 28]. The stability of the model is estimated according to the eigenvalue of the Jacobian matrix A, which is numerically computed in the following equation:

$$
\Delta \mathbf{X}^{k+1}=\mathbf{X}^{k+1}-\left.\mathbf{X}^{*} \approx \frac{\partial \mathbf{P}(\mathbf{X})}{\partial \mathbf{X}}\right|_{\mathbf{X}^{*}} \cdot \Delta \mathbf{X}^{k}=\mathbf{A} \cdot \Delta \mathbf{X}^{k}
$$

Equation (10) indicates that the disturbance $\Delta \mathbf{X}$ of the fixed point $\mathbf{X}$ in the stride-to-stride mapping leads to a deviation in the next stride through the Matrix A. The eigenvalue of the Jacobian matrix $\mathbf{A}$ is important. If there is an eigenvalue larger than 1 , the disturbance will accumulate over the time and the dynamic motion will leave the limit cycle finally. On the contrary, the motion with all eigenvalues smaller than 1 will makes the disturbance eliminated and keeps stable by its own self-stability. Therefore, the maximum magnitude of the eigenvalues $\max (\|\operatorname{eig}(\mathbf{A})\|)$ is used as the metric to assess the self-stability behavior.

Second, the ground reaction force (GRFs) is used as the metric to evaluate the compliance performance. The lower GRFs mean that for mammals or real quadruped robots, the interaction with the ground is compliant and the payload and damage on the leg are small. Through the comparison between two models, the effect of the spine on the compliance performance can be found.

2.6. Controller of the Model. It is known that although the passive model is able to achieve periodic motion, the motion is nominally stable [12]. When the eigenvalues of the motion are larger than 1, especially, the model cannot reject disturbances. To improve the stability of the model, a hybrid controller is used, as shown in Figure 3. The feedback control based on the virtual constraints [29] is applied for the control of the continuous states to analyze the effects of the coordination between the spinal joints on the motion stability. The linear quadratic regulator (LQR) [30] is applied for the control of the discrete states to adjust the attack angle at every half cycle. 


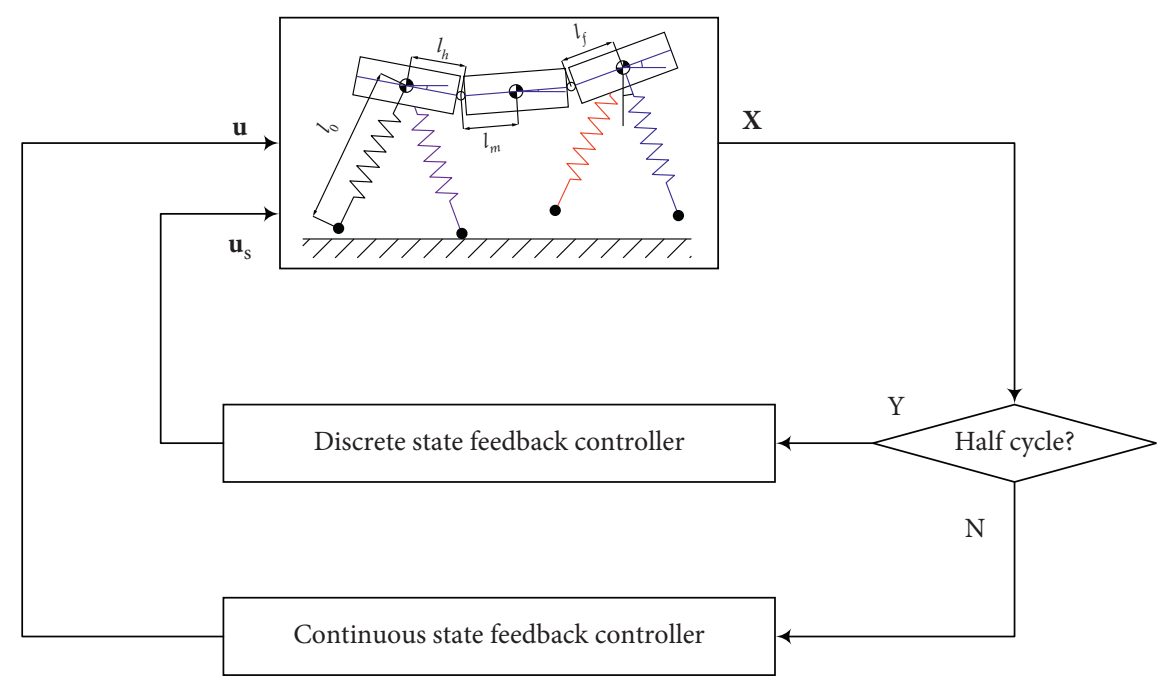

Figure 3: Control block diagram of the hybrid controller.

For the continuous states, the spinal joints of the model are chosen as the controlled joints, and the control object is to maintain the coordinated relation between the spine and the legs. The relationship between the generalized coordinates is a holonomic constraint that does not work on the model. Therefore, the output,

$$
\mathbf{y}=\mathbf{h}(\mathbf{q}):=\mathbf{H} \cdot \mathbf{q}-\mathbf{h}_{d}(\mathbf{q})
$$

is termed a virtual constraint that is required to be equal to zero [29], where $y$ indicates the angular output of two spinal joints which is the deviation of actual motion and expected motion. $\mathbf{H}$ is the mapping matrix from the generalized coordinates to the rotation angles of two spinal joints $\left(\theta_{f}=\varphi_{f}\right.$ $\left.-\varphi_{m}, \theta_{h}=\varphi_{m}-\varphi_{h}\right) . \mathbf{h}_{d}(q)$ indicates the expected rotation angles of the spinal joints that are restrained by the relationship with the generalized coordinates. Because $x$ is one of the generalized coordinates and is directly related to the leg motion, it is selected as the independent variable in the constraint. Importantly, $x$ is a monotone increasing variable. The expected rotation angles can be expressed as the $N$ th degree polynomial of $x$ :

$$
\mathbf{h}_{d i}(\mathbf{q})=\sum_{k=0}^{N} a_{i, k} s_{i}^{k}(\mathbf{q}), \quad i=f, h,
$$

where $a_{i, k}$ indicates the $k$ th coefficient of the $N$ th degree polynomial of front or hind spinal joint. The role of $s(\mathbf{q})$ is to transfer the function depending on time into the function depending on generalized coordinate $x$, which is expressed as follows:

$$
s(\mathbf{q})=\frac{x_{m}-x_{\text {cycle }}-x_{d, \text { min }}}{x_{d, \text { max }}-x_{d, \text { min }}},
$$

where $x_{d \text {, min }}$ and $x_{d \text {,max }}$ indicate the minimum and maximum displacement of the expected motion in half cycle. $x_{m}$ and $x_{\text {cycle }}$ indicate the current position and the position recorded at the half cycle event.

The expected motion is a nominal motion, which results from the passive motion without controller. The virtual constraint can be achieved with a feedback controller. That is, the function of the feedback controller is to guarantee the angular motion of the spinal joints and its coordination with the legs as the behavior that passive model shows. Therefore, the input actuators are set in parallel with the passive spinal joints. The control torque $\mathbf{u}$ is set as the following form:

$$
\left\{\begin{array}{l}
\mathbf{u}=\mathbf{u}^{*}+\mathbf{v}, \\
\mathbf{u}^{*}=-\left(L_{g} L_{f} h\right)^{-1} L_{f}^{2} h(\mathbf{x}), \\
\mathbf{v}=-\left(L_{g} L_{f} h\right)^{-1}\left(\mathbf{K}_{P} \cdot \mathbf{y}+\mathbf{K}_{D} \cdot \dot{\mathbf{y}}\right),
\end{array}\right.
$$

where $L_{g} L_{f} h$ and $L_{f}^{2} h$ are the Lie derivatives of the output function, which is computed from the second derivatives of the output function (11):

$$
\ddot{\mathbf{y}}=\underbrace{\left[\frac{\partial}{\partial \mathbf{q}}\left(\frac{\partial \mathbf{h}}{\partial \mathbf{q}} \cdot \dot{\mathbf{q}}\right) \frac{\partial \mathbf{h}}{\partial \mathbf{q}}\right] f(\mathbf{x})}_{L_{f}^{2} h}+\underbrace{\frac{\partial \mathbf{h}}{\partial \mathbf{q}} g(\mathbf{x})}_{L_{\mathrm{g}} L_{f} h} \mathbf{u} .
$$

By substituting the controller (14) into equation (15), the following equation can be obtained:

$$
\ddot{\mathbf{y}}+\mathbf{K}_{D} \dot{\mathbf{y}}+\mathbf{K}_{P} \mathbf{y}=0 .
$$

This equation is independent of the state space equations. $\mathbf{K}_{P}$ and $\mathbf{K}_{D}$ are the gain matrixes. Therefore, the system can be in the critical damping state when the gains meet $\mathbf{K}_{P}>0, \mathbf{K}_{D}=2 \sqrt{\mathbf{K}_{P}}$.

For the discrete states, due to the lack of dynamic effects of the legs during the swing phase, the attack angle $\mathbf{u}_{s}=\left[\alpha_{f}\right.$, $\left.\alpha_{h}\right]^{T}$ is the main control input, which is used to eliminate the deviations of the states. The Poincaré Mapping can be expressed as $\mathbf{X}(k+1)=\mathbf{P}\left(\mathbf{X}(k), \mathbf{u}_{s}(k)\right)$. By the differencing linear method, equation (10) is transferred into the following discrete form:

$$
\Delta \mathbf{X}(k+1)=\mathbf{A} \cdot \Delta \mathbf{X}(k)+\mathbf{B} \cdot \Delta \mathbf{u}_{s}(\mathbf{k}),
$$

where $\Delta \mathbf{X}=\mathbf{X}-\mathbf{X}^{*}$ indicates the controlled states. $\Delta \mathbf{u}_{s}=\mathbf{u}_{s}-\mathbf{u}_{s}^{*}$ indicates the control input of the discrete 
system. A and $\mathbf{B}$ are state matrix and control matrix, which are computed:

$$
\begin{aligned}
& \mathbf{A}=\left.\frac{\partial \mathbf{P}}{\partial \mathbf{X}}\right|_{X=X^{*}, u_{s}=u_{s}^{*}}, \\
& \mathbf{B}=\left.\frac{\partial \mathbf{P}}{\partial \mathbf{u}}\right|_{X=X^{*}, u_{s}=u_{s}^{*}} .
\end{aligned}
$$

According to the optimal control theory, the feedback control based on the discrete events is

$$
\Delta \mathbf{u}_{s}=-\mathbf{K} \Delta \mathbf{X}
$$

where $\mathbf{K}$ is the gain matrix of state feedback, which is computed by the Riccati equation. Therefore, the control input for discrete states is

$$
\mathbf{u}_{s}(k)=\mathbf{u}_{s}^{*}-\mathbf{K}\left(\mathbf{X}(k)-\mathbf{X}^{*}\right) \text {. }
$$

\section{Results and Discussion}

3.1. Motion of the Model with Two Spinal Joints. The dynamics characteristics of the model are investigated at different velocities taking $1 \mathrm{~m} / \mathrm{s}$ as the interval in the range $0.84 \mathrm{~m} / \mathrm{s} \sim 3.74 \mathrm{~m} / \mathrm{s}\left(F_{l}: 0.42 \sim 1.87\right)$. A large amount of simulation operations is carried out to find the suitable initial system parameters and state parameters to achieve the periodic motion at different velocities. As a result, the motion presents the same characteristics in the velocity range.

Figures 4 and 5, respectively, show the dynamics characteristics and the leg behavior of the periodic motion at $2.44 \mathrm{~m} / \mathrm{s}\left(F_{l}: 1.22\right)$. It is found that the springy legs in the model experience a process of compression and extension like the behavior of the SLIP model [2]. The feature is not changed even though the spine is added. As a result, the spine coordinated with the springy legs according to a specific dynamics characteristic. The ground reaction forces and the gait sequence of four legs presented in Figures 5(a) and 5(d) meet the features of the trotting motion, in which the diagonal legs nearly move simultaneously.

In addition, through a large amount of simulations at different velocities, there is an interesting phenomenon that the rotation amplitude of the spinal joints has nothing to do with the set velocity, as shown in Figure 6. During the stance phase, the front and hind body rotate at opposite direction aiming at dropping the barycenter of the middle body. During the swing phase, the front and hind body rotate with a smaller amplitude at opposite direction aiming at raising the barycenter of the middle body. This is a motion pattern accompanied with the oscillation of barycenter of the middle body.

The finding in the simulation can be explained according to the function of the spine at trotting gait. The biological research that studies the function of spinal muscle in different gaits reveals that when the motion at trotting gait has larger motion acceleration, the muscle in the spine will be induced to generate the bending motion in the sagittal plane, which is used to balance the forces and bigger vertical oscillation. In the simulation process, the suitable stiffness leads to the normal motion. It means that the motion pattern of the spinal joints and corresponding elastic torque can not only stabilize the motion oscillation, but also balance the forces in the springy legs.

3.2. Dynamics Characteristics at Different Velocities. The stiffness of the spinal joints and legs, the attack angle of the legs, and the vertical displacement of the body in the solution space are chosen to investigate the changing of the dynamic features of the periodic motion at different initial velocity.

In Figures 7(a) and 7(b), the required stiffness of the spinal joints and the legs at different velocities are recorded. It is found that no matter at which velocity the model is moving, the stiffness of the front spinal joint is larger than that of the hind spinal joint. The stiffness of the hind joint nearly keeps constant at different velocities and the stiffness of the front joint has an increasing tendency with the velocity increasing. It is similar to the structure feature of mammals. The thoracic vertebra has a hard and straight structure to protect the internal organs, and the lumbar vertebra possesses a soft structure to bend to adapt to different gaits [31]. It is regarded that the soft feature of the hind spinal joint is ensured with the constant small stiffness. The changing of the stiffness of the front spinal joint means that a larger stiffness is needed to increase the output torque to balance the inertia effect caused by the increasing velocity. It also coordinates with the stiffness changing of the legs to adapt to a higher speed locomotion.

The stiffness characteristic of the legs shown in Figure $7(\mathrm{~b})$ is consistent with the fact that at a higher velocity, the leg with a relative low stiffness [32] allows for ensuring compliance and absorbing the large kinetic energy into the potential energy, which eliminates the possibility of occurring of high frequency oscillation and reduces the damage on the legs. In addition, the matching between the stiffness of front spinal joint and hind spinal joint is also used to coordinate with the legs in order to counteract the effect of ground reaction forces on the body stability. Therefore, it is suggested that the suitable stiffness combination of two spinal joints and four legs should be established to generate the self-stable periodic trotting motion for the model $[12,14]$.

It is found in Figure 7 (c) that with the velocity increasing, the vertical displacement decreases. It means that when the legs are lifted off the ground the angle relative to the vertical direction is bigger at a higher speed. Meanwhile, the attack angle increases (Figure $7(\mathrm{~d})$ ). As a result, to obtain the periodic motion with a higher speed, the bigger stride length and swing range of the leg are necessary for the model with two spinal joints. Overall, the model with two spinal joints is able to be used as the template of the quadruped trotting motion and to reflect the coordination between the spine and the legs.

3.3. Comparison between Two Models. The eigenvalue of the Jacobian matrix represents the self-stable feature of the periodic motion when the motion states of the model produce some deviations caused by other disturbances. In 


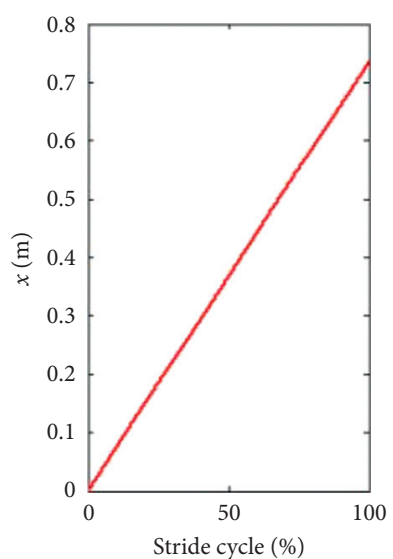

(a)

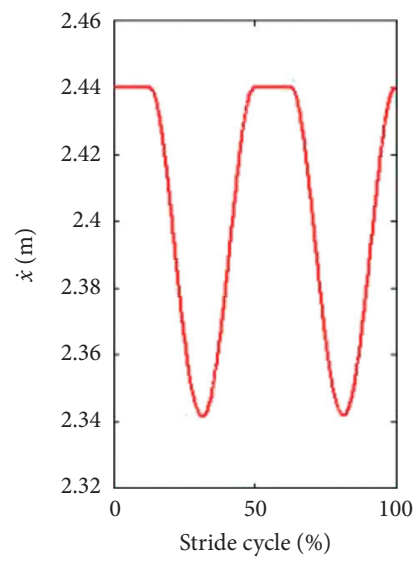

(c)

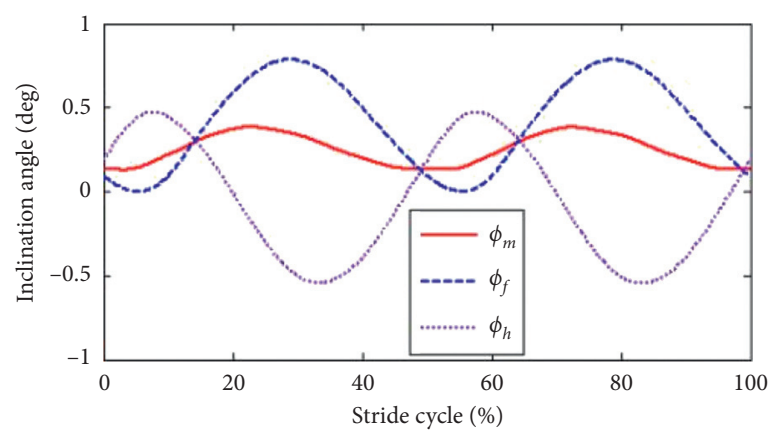

(e)

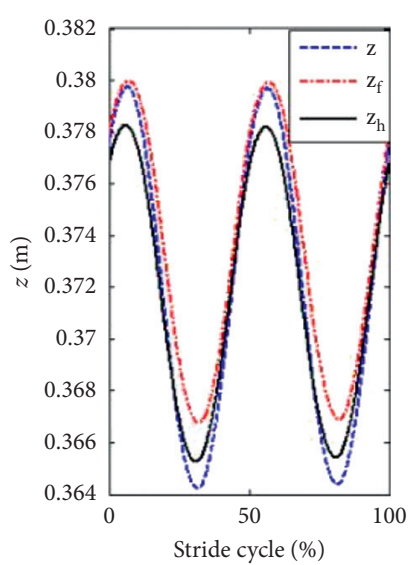

(b)

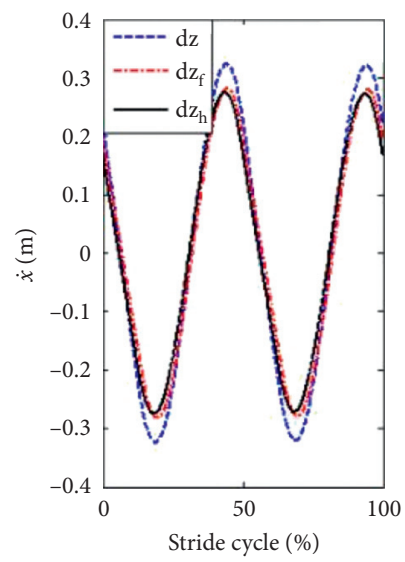

(d)

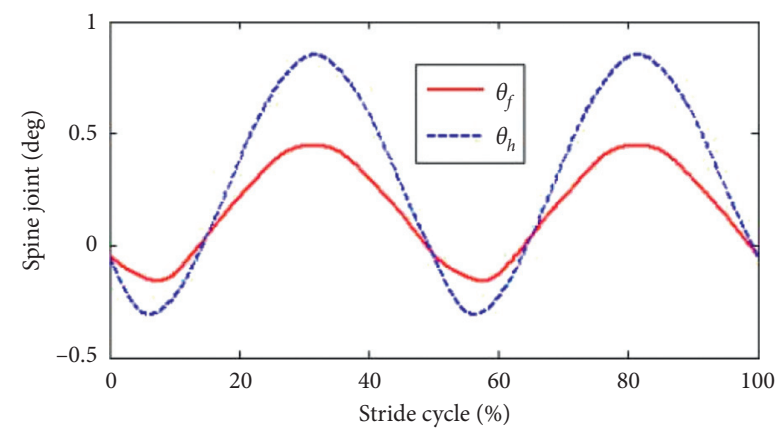

(f)

Figure 4: Dynamic behavior of the model with two spinal joints at $2.44 \mathrm{~m} / \mathrm{s}$. (a) Horizontal displacement of middle body, (b) vertical displacement of three bodies, (c) horizontal velocity of middle body, (d) vertical velocity of three bodies, (e) inclination angle of three bodies, and (f) the rotation angle of two spinal joints.

Figure $8(a)$, it is found that the variation tendencies of the eigenvalues of the two models are almost the same. The difference of the values is also small. That means compared with the model with rigid body, the added spine has no obvious effects on the local stability which is numerically computed to reflect the sensibility to the initial small perturbations. The two models possess the same velocity range $(2.14 \mathrm{~m} / \mathrm{s}-2.64 \mathrm{~m} / \mathrm{s})$ in which the maximum magnitude of the eigenvalues is smaller than 1 . In addition, it is worth noting that the starting point of the velocity area whose eigenvalue is smaller than 1 is close to the Froude number $\left(2 \mathrm{~m} / \mathrm{s}, F_{l}: 1\right)$ at which the mammals transform from the walking gait to trotting gait.

Figure 8(b) shows the peak ground reaction forces of forelimb and hindlimb of the two models. It is found that the peak GRFs of the model with two spinal joints are all smaller than those of the model with rigid body in the range of Froude numbers of Canine's trotting gait $\left(F_{1}: 1 \sim 2.2\right)$. Furthermore, it is interesting that reduction is more obvious in the range where the eigenvalues are smaller than 1 . The maximum 


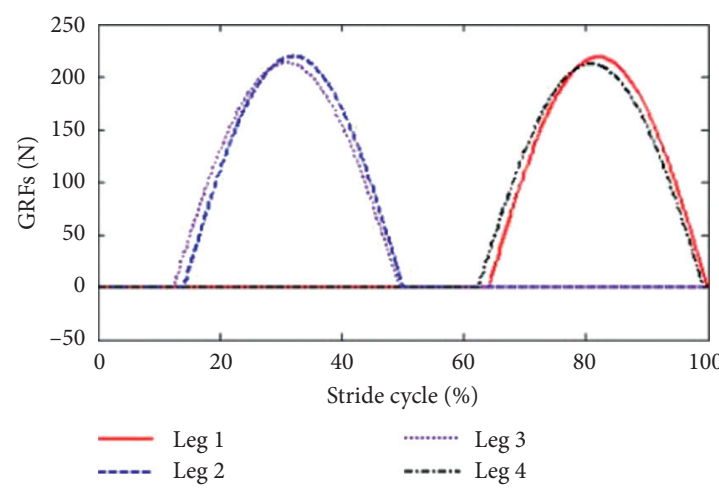

(a)

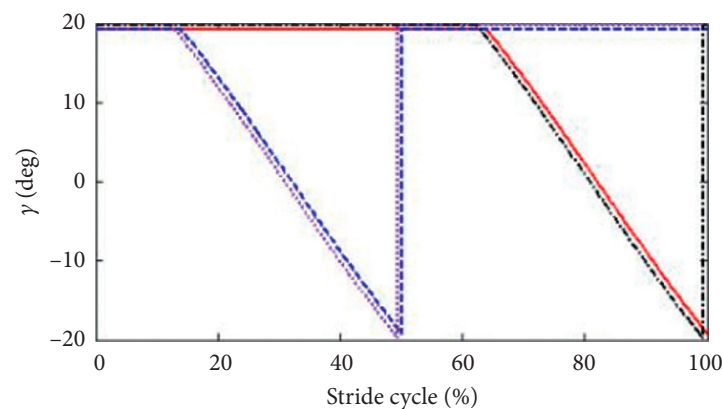

(c)

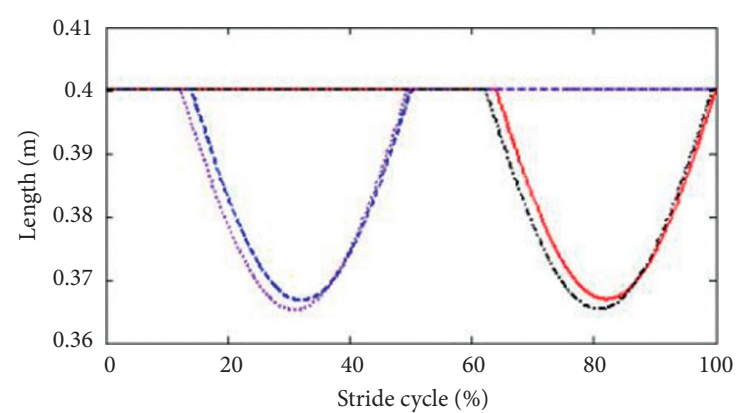

(b)

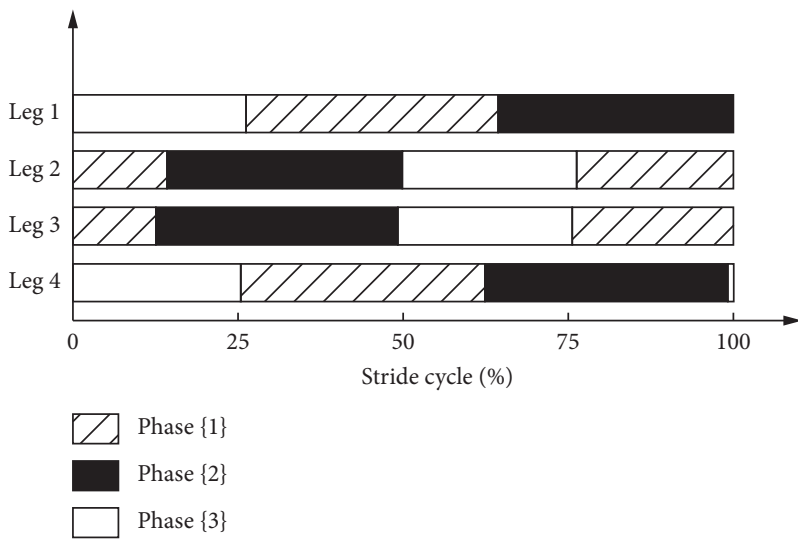

(d)

FIGURE 5: Leg behavior and gait sequence at $2.44 \mathrm{~m} / \mathrm{s}$. (a) Ground reaction forces of four legs, (b) length change of four springy legs, (c) elevation angle of four legs, and (d) gait feature.

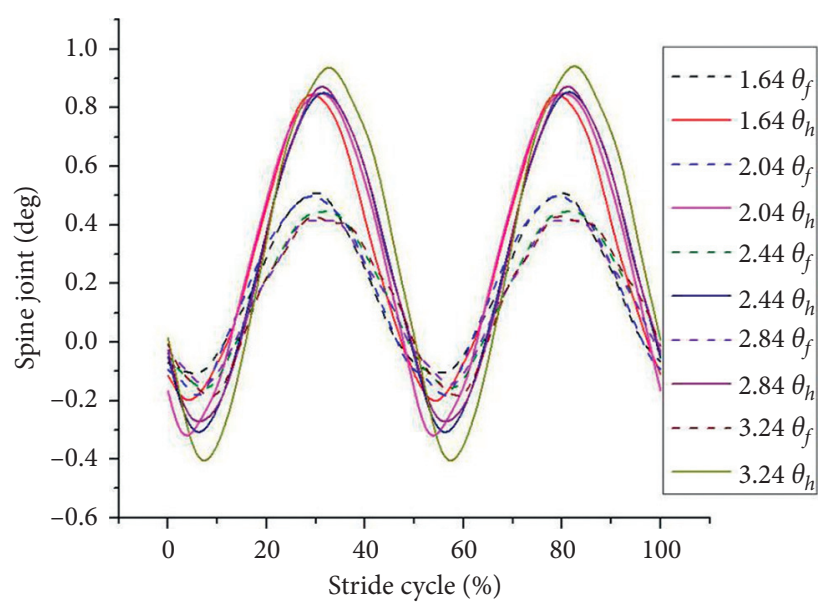

FIGURE 6: Motion of spinal joints at different velocities.

reduced amplitude of the peak forces can reach $90 \mathrm{~N}$. As a result, the added spine has an important effect on the trotting motion. It has the function of reducing the ground reaction forces and improving the compliant interaction with the ground. In addition, it is found that when the velocity is higher than $2.64 \mathrm{~m} / \mathrm{s}$, both the stiffness and GRFs of the forelimb will be bigger than those of the hindlimb.
3.4. Control for the Model at Trotting Gait. As described above, the periodic trotting motion of the model with two spinal joints is nominal. Due to the existence of the numerical errors in the initial states, the model will lose stability if the maximum eigenvalue is bigger than 1 at one velocity. Figure 9 shows the feature of the ground reaction forces imposed on the passive model at $1.84 \mathrm{~m} / \mathrm{s}$. During three 


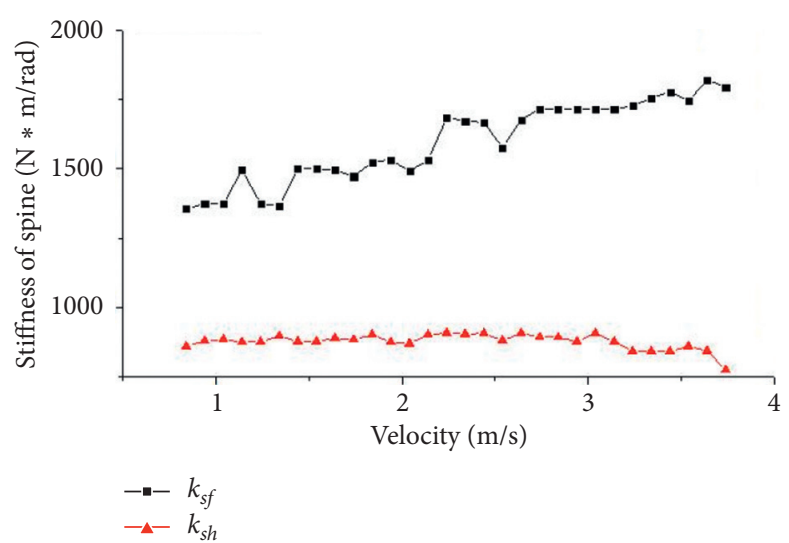

(a)

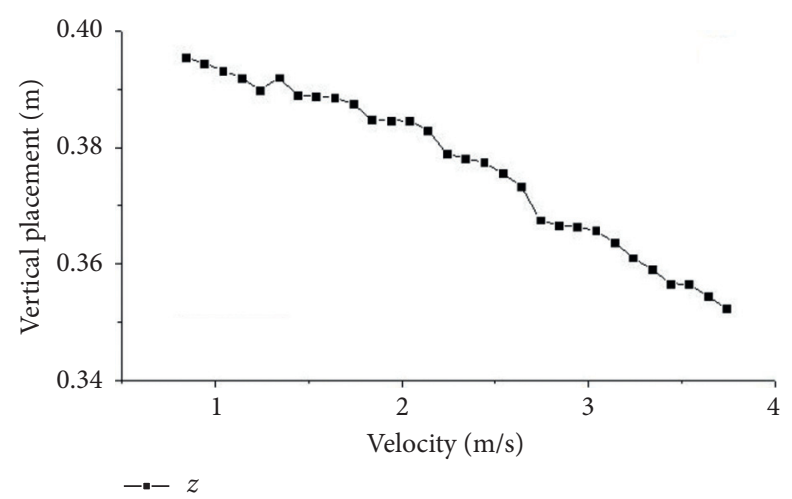

(c)

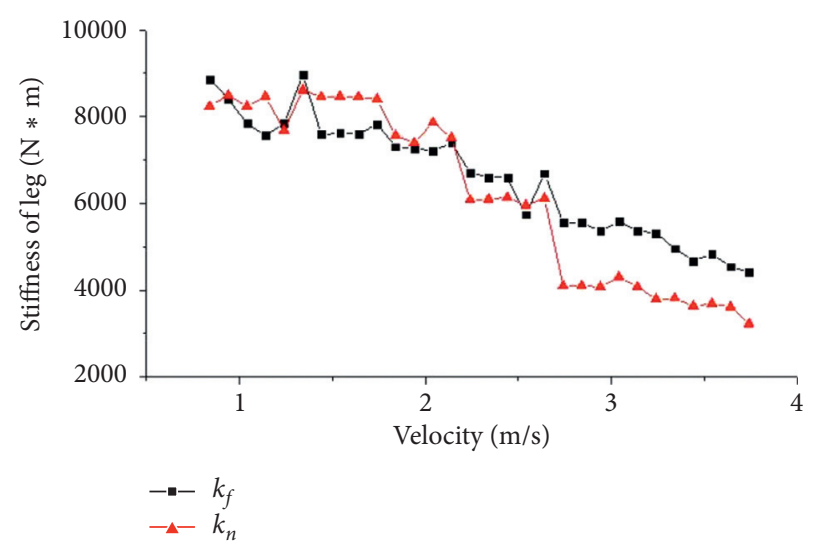

(b)

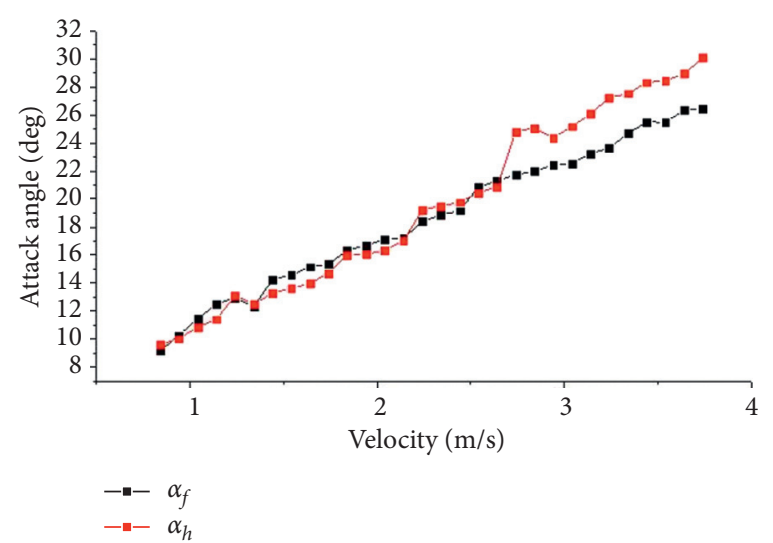

(d)

Figure 7: Dynamics characteristics at different velocities. (a) Stiffness of front and hind spinal joint, (b) stiffness of forelimb and hindlimb, (c) initial vertical displacement of the middle body, and (d) attack angle of forelimb and hindlimb.

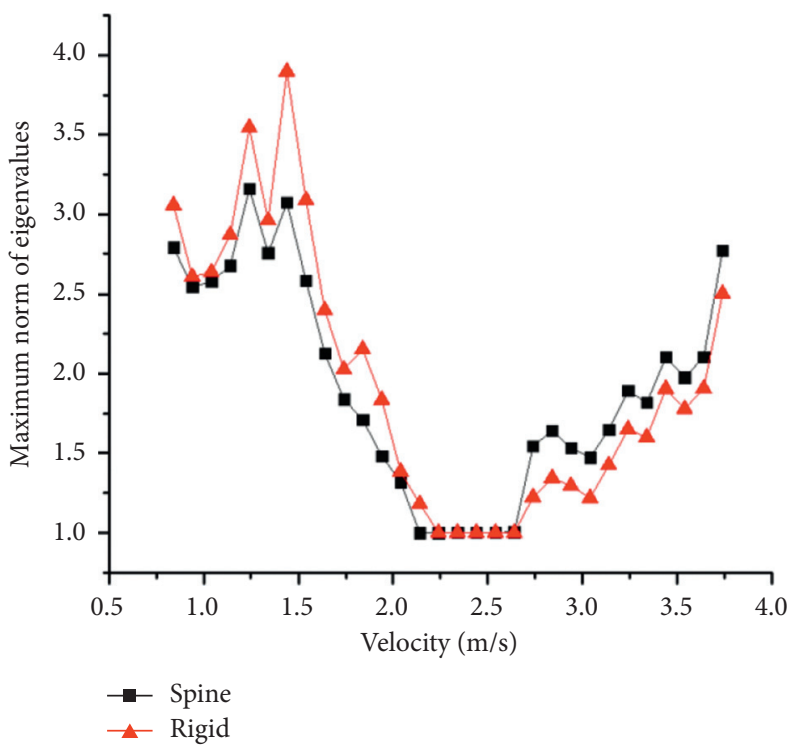

(a)

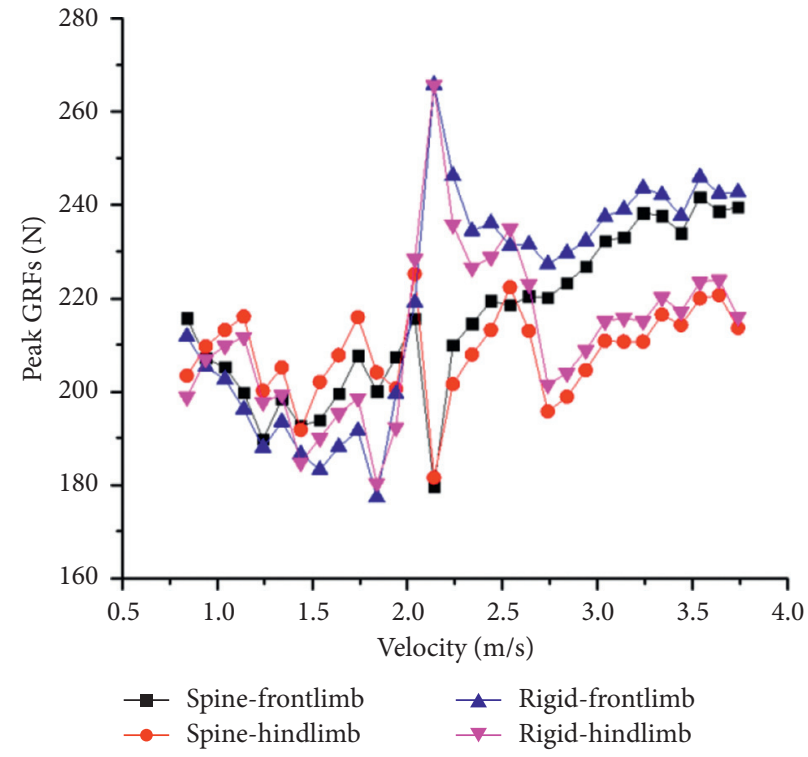

(b)

FIGURE 8: Eigenvalue and GRFS of model with and without spine at different velocities. (a) Maximum magnitude of the eigenvalues, and (b) peak ground reaction forces of forelimb and hindlimb. 


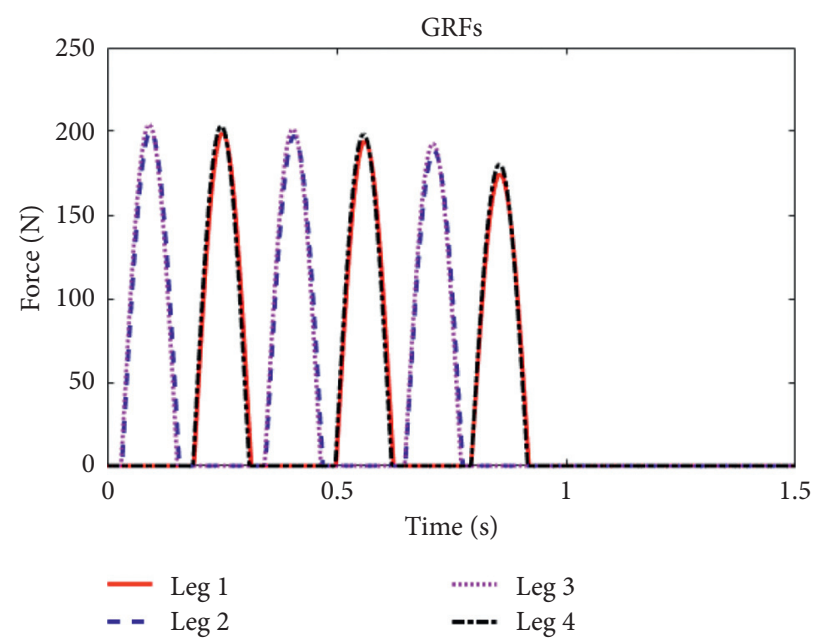

Figure 9: GRFs of the passive model at $1.84 \mathrm{~m} / \mathrm{s}$.

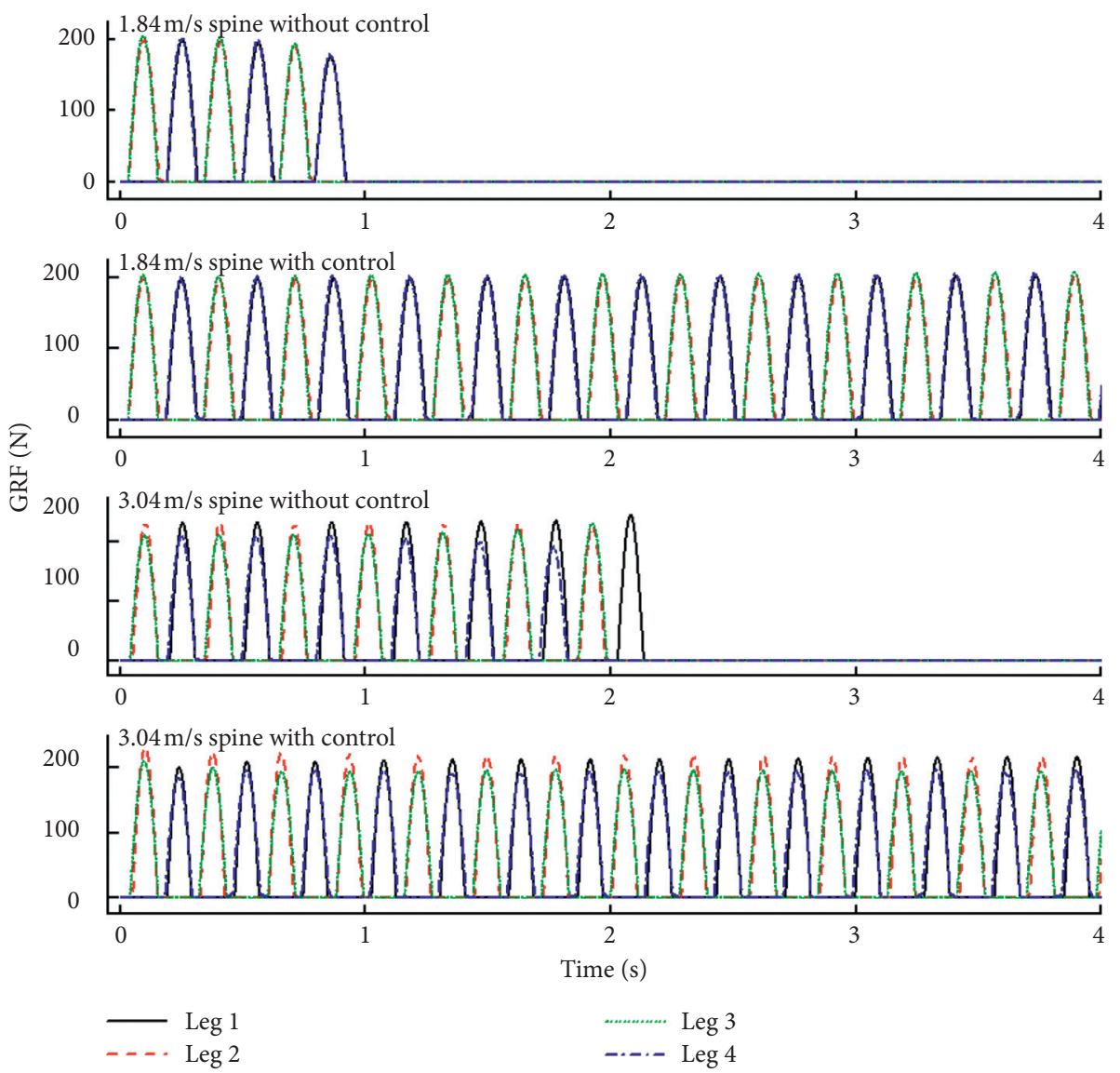

FIgURE 10: Ground reaction forces of the models when they move at low speed $(1.84 \mathrm{~m} / \mathrm{s})$ and high speed $(3.04 \mathrm{~m} / \mathrm{s})$.

cycles of motion, the model loses the support of the legs and falls down because of the accumulation of the errors.

Figure 10 shows the ground reaction forces of the models when they move at low speed and high speed, which include the speeds whose maximum eigenvalues are bigger than 1 . It is found that they all fall down when the control has not been added. Furthermore, the bigger the maximum eigenvalue is, the more quickly the model loses stability. While the control input whose purpose is to ensure the motion coordination is applied into the model, the model will adjust the motion state through a few cycles. Then, the model will enter the stable pathway. Meanwhile, the ground reaction forces reach 


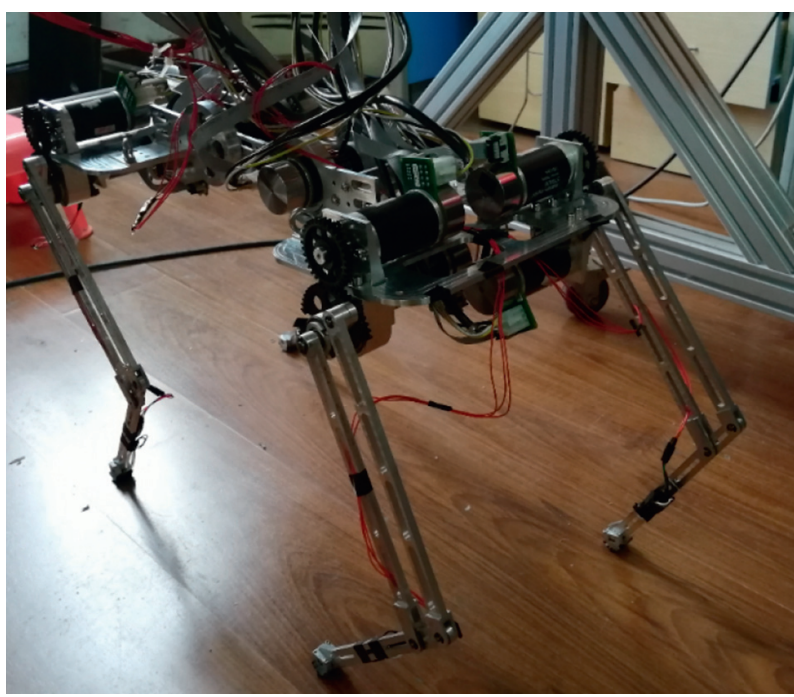

FIgURE 11: Quadruped robot with two spinal joints.

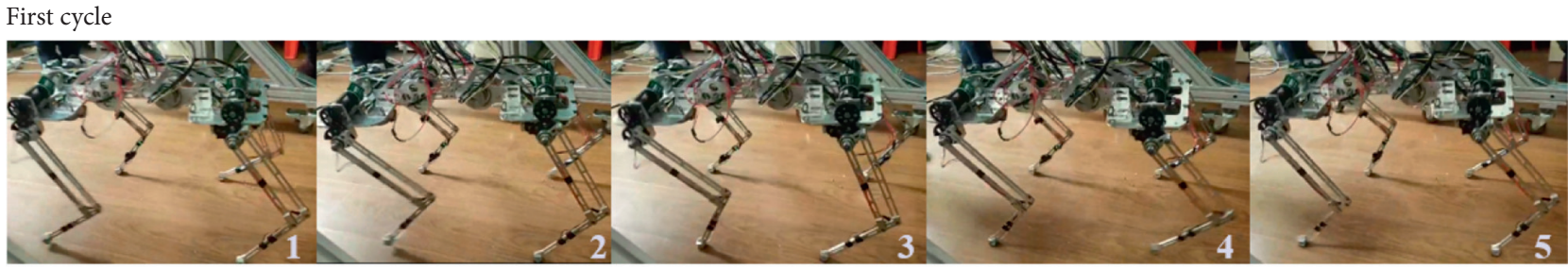

Second cycle

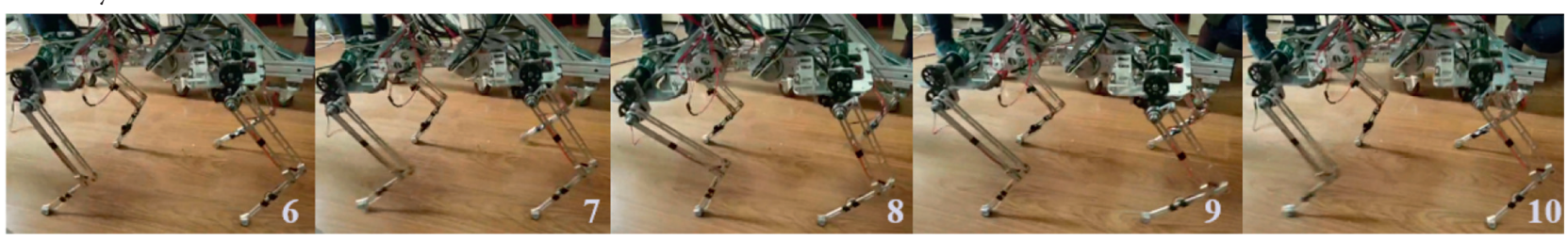

FIgURE 12: Trotting motion of the quadruped robot.

a stable state after a period of fluctuation. This means that the coordination between the spine and leg contribute to the stable motion.

3.5. Quadruped Robot Based on the Model. The above results explain the effects of the spine on improving the performance of the quadruped motion during the trotting gait. It contributes to guide the design and development of the quadruped robot. Hence, the physical prototype of the quadruped robot is designed and established based on the quadruped model, as shown in Figure 11. It has two compliant spinal joints which is achieved by compliant control methods. The legs have two freedoms, hip joint and knee joint. The knee joint is actuated by the motor through the link mechanism. Therefore, the motors are all placed on the body. Through the gait planning and motion control, the trotting motion is achieved, as shown in Figure 12. It means that the quadruped robot with two spinal joints is able to move at trotting gait.
In order to demonstrate the effect of the two spinal joints on the motion performance as described above. The comparative experiments are carried out. In the experiments, the quadruped robot is controlled in two modes. Five groups of experiments in which the quadruped robot is able to maintain steady periodic motion are carried out and recorded. The ground reaction forces of legs are collected by the force sensor in the foot, and the torque of the leg joint is computed according to the motor torque and the reduction ratio. In these two modes, the legs are all controlled by the impedance control methods. In order to reduce the differences between these two modes, the structure of the quadruped robot is maintained invariant. The quadruped robot possesses two spinal joints. The only difference is the applied control method. In the first mode, the spinal joints are controlled by position control methods to maintain the fixed position, which is considered as the "rigid" spine due to the 'stiff' feature of position control. In the second mode, the spinal joints are controlled by the compliant control methods. In the reality motion of the quadruped robot, the 
TABLE 2: Peak ground reaction forces and torque by two control modes.

\begin{tabular}{|c|c|c|c|c|c|}
\hline \multirow{2}{*}{ Control mode } & & \multicolumn{2}{|c|}{ First mode } & \multicolumn{2}{|c|}{ Second mode } \\
\hline & & Mean & $\mathrm{SD}$ & Mean & $\mathrm{SD}$ \\
\hline \multirow{2}{*}{ Peak GRF (N) } & Front leg & 90.9 & 9.0 & 82.3 & 14.6 \\
\hline & Hind leg & 73.0 & 15.3 & 59 & 18.1 \\
\hline \multirow{4}{*}{ Peak torque of leg joints $(\mathrm{N} * \mathrm{~m})$} & Front hip & 12.2 & 0.7 & 11.8 & 1.0 \\
\hline & Front knee & 14.6 & 0.4 & 13.8 & 0.5 \\
\hline & Hind hip & 16.2 & 1.9 & 15.0 & 1.8 \\
\hline & Hind knee & 15.0 & 1.0 & 15.4 & 1.4 \\
\hline
\end{tabular}

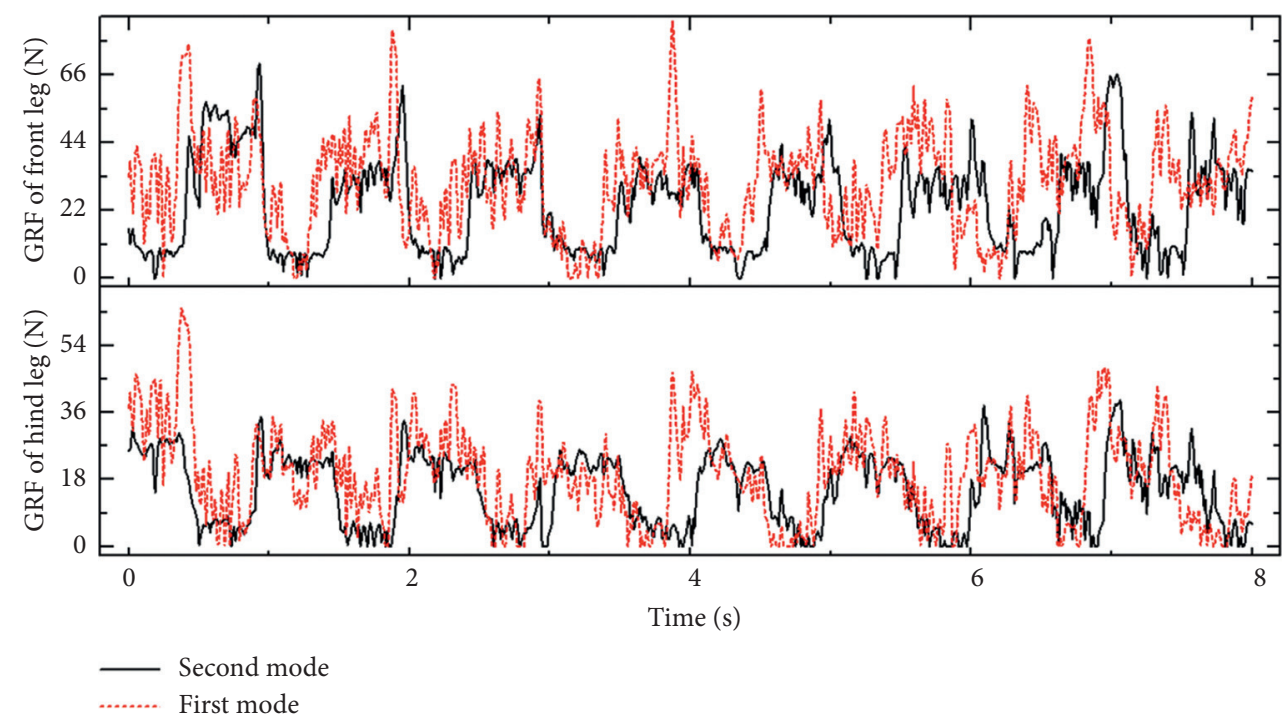

Figure 13: Ground reaction forces of the quadruped robot by two control modes.

spinal joints show very small amplitude of motion and make the robot keep compliant interaction with the ground by the output compliant torque.

Table 2 shows the peak ground reaction forces and the peak torque of the leg joint in the experiments, in which the mean indicates the average value of five groups, and the SD indicates the standard deviation of these experimental sample data. Figure 13 shows the curves of the ground reaction forces when the quadruped robot controlled by two modes moves in one group of these experiments. The red dashed line and black solid line indicate the first mode and second mode, respectively. It is found from the table and figure that the peak ground reaction forces of the legs show obvious decline, which meant that when the compliant spinal joints are added, the interaction between the legs with the ground is improved. In addition, it is found that the torque of the leg joints has a small range of reduction when the compliant control is applied into the spinal joints. Hence, the comparative experiments reflect the effect of the spinal joints on the motion performance, especially the interaction with the ground.

\section{Conclusions}

In this paper, a model with two passive spinal joints is proposed to study the effects of the spine motion on the performance of the quadruped trotting gait. It reveals that the quadruped trotting motion is able to be described by this model because of the similarity of the feature of the gait and stiffness to the mammal. It is suggested that the suitable stiffness combination of the spinal joints and legs plays an important role in the periodic motion creation. There is an interesting finding that the spinal joints have specific motion at different velocities. By comparing with the spine motion of the model with rigid body, it is found that although the rotation angle of the spinal joints is very small, the spinal joints capture important influences on stabilizing the forces and making the interaction with the ground more compliant in a special velocity range. In addition, a hybrid controller is added and replaced the passive spinal joints in the model. The results reveal that the control based on the kinematic coordination between the spinal joints can eliminate the initial perturbation and obtain a real stable periodic motion.

This study not only helps us to have a good understanding of the coordination between the spinal joints but also comprehends the effects of the spine on the trotting motion. Hence, the prototype of the quadruped robot with two spinal joints was established, and the trotting motion was also achieved. Finally, the experiments of quadruped robot demonstrate the effect of the spinal joints on the motion performance, especially the interaction with the ground. Furthermore, the simulation results contribute to 
expanding the model to a large range of gaits and guiding us to developing the quadruped robots which can move at a large range of velocities and gaits. In the future, the dynamic analysis and the motion control of the quadruped robot will be further carried out.

\section{Data Availability}

The data used to support the findings of this study are available from the corresponding author upon request.

\section{Conflicts of Interest}

The authors declare that there are no conflicts of interest regarding the publication of this paper.

\section{Acknowledgments}

This work was supported by the National Natural Science Foundation of China (Grant no. 51705382).

\section{References}

[1] F. Lacquaniti, R. Grasso, and M. Zago, "Motor patterns in walking," News in Physiological Sciences, vol. 14, no. 1, pp. 168-174, 1999.

[2] M. H. Dickinson, C. T. Farley, R. J. Full et al., "How animals move: an integrative view," Science, vol. 288, no. 5463, pp. 100-106, 2000.

[3] R. Blickhan, "The spring mass model for running and hopping," Journal of Biomechanics, vol. 22, no. 11-12, pp. 1217-1227, 1989.

[4] M. M. Ankaralı and U. Saranll, "Analysis and control of a dissipative spring- mass hopper with torque actuation," Robotics: Science \& Systems Vi, 2010.

[5] H. E. Orhon, C. Odabas, I. Uyanik et al., "Extending the lossy spring-loaded inverted pendulum model with a slider-crank mechanism," in Proceedings of the the 17th International Conference on Advanced Robotics, Istanbul, Turkey, July 2015.

[6] G. Garofalo, C. Ott, and A. Albu-Schaffer, "Walking control of fully actuated robots based on the bipedal SLIP model," in Proceedings of the IEEE International Conference on Robotics and Automation, Saint Paul, MN, USA, May 2012.

[7] Z. Li, Y. Tan, S. Zeng, and H. Y. Luo, "Dynamics analysis and planning for a specific leg model with A variable stiffness element," International Journal of Simulation Modelling, vol. 4, no. 16, pp. 707-719, 2017.

[8] P. Nanua and K. J. Waldron, "Energy comparison between trot, bound, and gallop using a simple model," Journal of Biomechanical Engineering, vol. 117, no. 4, pp. 466-473, 1995.

[9] M. Hildebrand, "Motions of the running cheetah and horse," Journal of Mammalogy, vol. 4, no. 40, pp. 481-495, 1959.

[10] R. M. Alexander, N. J. Dimery, and R. F. Ker, "Elastic structures in the back and their role in galloping in some mammals," Journal of Zoology, vol. 207, no. 4, pp. 469-482, 2009.

[11] B. M. Haueisen, Investigation of an Articulated Spine in a Quadruped Robotic System, The University of Michigan, Ann Arbor, MI, USA, 2011.

[12] Q. Cao and I. Poulakakis, "Quadrupedal bounding with a segmented flexible torso: passive stability and feedback control," Bioinspiration \& Biomimetics, vol. 8, no. 4, p. 46007, 2013.
[13] Q. Cao and I. Poulakakis, "On the energetics of quadrupedal running: predicting the metabolic cost of transport via a flexible-torso model," Bioinspiration \& Biomimetics, vol. 10, no. 5, p. 56008, 2015.

[14] Q. Deng, S. Wang, W. Xu, J. Mo, and Q. Liang, "Quasi passive bounding of a quadruped model with articulated spine," Mechanism and Machine Theory, vol. 52, pp. 232-242, 2012.

[15] X. Wei, Y. Long, C. Wang, and S. Wang, "Rotary galloping with a lock-unlock elastic spinal joint," Proceedings of the Institution of Mechanical Engineers, Part C: Journal of Mechanical Engineering Science, vol. 229, no. 6, pp. 1088-1102, 2014.

[16] W. Z. Li, B. R. Wang, and D. J. Chen, "Gait planning for quadruped robot with parallel spine," Journal of Zhejiang University (Engineering Science), vol. 52, no. 7, pp. 1267-1274, 2018.

[17] Z. Wei, G. M. Song, and H. Y. Shun, "Kinematic modeling and trotting gait planning for the quadruped robot with an active spine," Journal of Southeast University (Natural Science Edition), vol. 49, no. 6, pp. 1019-1025, 2019.

[18] K. Weinmeister, P. Eckert, H. Witte, and A. J. Ijspeert, "Cheetah-cub-S: steering of a quadruped robot using trunk motion," in Proceedings of the 2015 IEEE International Symposium on Safety, Security, and Rescue Robotics (SSRR), West Lafayette, IN, USA, October 2015.

[19] Q. Zhao, H. Sumioka, X. Yu, K. Nakajima, Z. Wang, and R. Pfeifer, "The function of the spine and its morphological effect in quadruped robot locomotion," in Proceedings of the 2012 IEEE International Conference on Robotics and Biomimetics, Guangzhou, China, December 2012.

[20] C. D. Remy, K. Buffinton, and R. Siegwart, "MATLAB framework for efficient gait creation," in Proceedings of the International Conference on Intelligent Robots and Systems, San Francisco, CA, USA, September 2011.

[21] T. Mcgeer, "Passive dynamic walking," The International Journal of Robotics Research, vol. 9, no. 2, pp. 62-82, 1990.

[22] J. J. Robilliard, T. Pfau, and A. M. Wilson, "Gait characterisation and classification in horses," Journal of Experimental Biology, vol. 210, no. 2, pp. 187-197, 2007.

[23] M. H. Raibert, "Running with symmetry," The International Journal of Robotics Research, vol. 5, no. 4, pp. 3-19, 1986.

[24] M. Clerc and J. Kennedy, "The particle swarm-explosion, stability, and convergence in a multidimensional complex space," IEEE Transactions on Evolutionary Computation, vol. 6, no. 1, pp. 58-73, 2002.

[25] S. Pouya, M. Khodabakhsh, A. Spröwitz, and A. Ijspeert, "Spinal joint compliance and actuation in a simulated bounding quadruped robot," Autonomous Robots, vol. 41, no. 2, pp. 437-452, 2017.

[26] R. M. Alexander, "The gait of bipedal and quadrupedal animals," The International Journal of Robotics Research, vol. 3, no. 2, pp. 49-59, 1984.

[27] C. D. Remy, K. Buffinton, and R. Siegwart, "Stability analysis of passive dynamic walking of quadrupeds," The International Journal of Robotics Research, vol. 29, no. 9, pp. 1173-1185, 2010.

[28] J. H. Dong, O. S. Sang, J. Lee, and S. Kim, "High speed trotrunning: implementation of a hierarchical controller using proprioceptive impedance control on the MIT cheetah," International Journal of Robotics Research, vol. 33, no. 11, pp. 1417-1445, 2014.

[29] E. R. Westervelt, J. W. Grizzle, C. Chevallereau et al., Feedback Control of Dynamic Bipedal Robot Locomotion, Taylor \& Francis/CRC Press, Boca Raton, FL, USA, 2007. 
[30] E. Todorov, Optimal Control Theory, Springer US, New York, NY, USA, 2000.

[31] N. Schilling and D. R. Carrier, "Function of the epaxial muscles in walking, trotting and galloping dogs: implications for the evolution of epaxial muscle function in tetrapods," Journal of Experimental Biology, vol. 213, no. 9, pp. 1490-1502, 2010.

[32] H. M. Herr and T. A. Mcmahon, "A trotting horse model," The International Journal of Robotics Research, vol. 6, no. 19, pp. 566-581, 2000. 